\title{
双臂协调机器人相对动力学建模
}

\author{
张建华 ${ }^{1,2}$ 许晓林 ${ }^{1}$ 刘 璇 ${ }^{1}$ 张明路 $^{1}$ \\ (1. 河北工业大学机械工程学院 天津 300130; \\ 2. 中国科学院沈阳自动化研究所机器人学国家重点实验室 沈阳 110016)
}

\begin{abstract}
摘要: 双臂机器人动力学建模是研究双臂协调运动的关键技术, 难点在于同一系统中建立两机械臂之间联系。针对双臂协调 机器人动力学建模问题, 基于拉格朗日方程建立了两臂动力学模型一般形式, 运用矢量解析法求解单臂角速度雅可比矩阵, 然后结合虚位移原理, 基于相对雅可比矩阵建立双臂协调机器人相对动力学模型, 确立两机械臂末端相对作用力与关节参数 之间关系。仿真和试验结果验证了矢量解析法与相对动力学模型的正确性, 该模型能够求解两机械臂末端相对力, 为分析双 臂协调运动提供理论依据。
\end{abstract}

关键词: 相对雅可比矩阵; 协调; 动力学建模; 双臂机器人

中图分类号: TH113

\section{Relative Dynamic Modeling of Dual-arm Coordination Robot}

\author{
ZHANG Jianhua $^{1,2}$ XU Xiaolin ${ }^{1} \quad$ LIU Xuan $^{1} \quad$ ZHANG Minglu $^{1}$ \\ (1. School of Mechanical Engineering, Hebei University of Technology, Tianjin 300130; \\ 2. State key Laboratory of Robotics, Shenyang Institute of Automation, \\ Chinese Academy of Sciences, Shenyang 110016)
}

\begin{abstract}
The dynamic modeling of dual-arm robot is the key technology to study the coordinated motion of the two arms. The difficulty lies in establishing the connection between the two robot arms in the same system. Aiming at the problem of dynamic modeling of dual-arm coordination robot, the general dynamic models of the two manipulators based on the Lagrangian equation is established, and the vector analytical method is used to solve the angular velocity Jacobian matrix of the single arm. Then combining the principle of virtual displacement, and based on the relative Jacobian matrix, the relative dynamics model of the dual-arm coordination robot is established. And the relationship between the relative forces of the ends of the two manipulators and the joint parameters is established. Simulation and experimental results demonstrate the correctness of vector analytical method and relative dynamics model. The model can solve the relative force of the ends of the two manipulators and provide a theoretical basis for analyzing the coordinated motion of the two arms.
\end{abstract}

Key words: relative Jacobian matrix; coordination; dynamics modeling; dual-arm robot

\section{0 前言}

随着机器人技术的发展, 仿人双臂机器人协调 运动的研究日益增多。双臂机器人不是两个单臂机 器人简单叠加, 而是在同一系统中相互配合、相互 联系, 故双臂协调动力学模型是研究双臂机器人装 配、搬运、加工等复杂操作的核心关键技术。

目前, 双臂协调动力学模型主要有两种: 一是

* 国家自然科学基金(51575157, 61473113)、机器人学国家重点实验室基金 (2015002)、河北省自然科学基金重点(E2016202342)和智能机器人与系 统高精尖创新中心开放基金（2018JRS04）资助项目。20180814 收到初 稿, 20181102 收到修改稿
两臂捕捉目标物体形成闭链系统的研究。利用目标 物体与机械臂之间相互作用力形成闭环约束关系, 建立目标物体与机械臂的联系, 主要分析夹持物体 运动的作用力和夹持内力。 $\mathrm{TAO}$ 等 ${ }^{[1-3]}$ 分别建立平面 双臂及被捕获目标的动力学方程, 再利用闭链系统 的闭环约束关系, 获得闭链混合体系统动力学方程。 $\mathrm{JIA}$ 等 ${ }^{[4-5]}$ 利用凯恩方程建立双臂动力学模型, 推导 其与目标物体的约束关系建立闭链模型。刘佳等 ${ }^{[6]}$ 基于 Udwadia-Kalaba 方程建模思想, 克服传统拉格朗 日方程需借助拉格朗日乘子求解动力学方程的缺点, 先建立系统不受约束的动力学方程, 再根据假设情 况建立约束方程, 联合建立完整的双臂动力学模型。 综上, 双臂协调机器人的动力学建模局限于平面模 
型和两臂夹持目标物体, 依靠物体与机械臂的约束 关系，求解两机械臂与目标物体的闭链系统模型。

二是两机械臂末端相对力的研究。将双臂末端 的相对运动视为单机械臂的运动, 基于单机械臂动 力学模型求解两机械臂末端绝对运动参数, 主要分 析两臂末端接触瞬间作用力与操作物体装配时接触 碰撞力, 目前相关研究较少, 建立的模型不完善。

JAMISOLA 等 ${ }^{[7]}$ 利用单臂动力学模型, 结合相对雅 可比矩阵将两机械臂独立的动力学融合到一起, 双 臂作为单机械臂处理, 视作一个整体, 建立双臂模 块化动力学模型。上述模型确立关节力矩与各关节 运动参数之间关系, 考虑末端执行器之间相对运动 设计相对力控制器, 然而没有推导两机械臂末端相 对力的相关模型。SHIN 等 ${ }^{[8]}$ 利用一种虚拟动力学模 型(VDM), 基于力和力矩(FT)传感器检测末端力, 使机器人能与环境进行物理交互, 控制双臂运动。 但是虚拟动力学模型无法求得机械臂末端作用力, 需要在手腕处安装 FT 传感器, 配合关节编码器获 取当前关节位置检测末端力。

为改进上述两种建模方法局限闭链约束和缺少 相对力模型的缺点, 本文提出采用相对动力学模型 的概念。首先建立空间六自由度双臂模型, 再利用 单臂雅可比矩阵求解双臂系统相对雅可比矩阵, 然 后结合虚位移原理, 推导两机械臂末端相对力表达 式, 完成双臂协调机器人相对动力学系统建模。然 而在求解单臂雅可比矩阵过程中, 利用传统微分变 换法和矢量积法 ${ }^{[9]}$ 都是构造法, 构造过程求解繁琐, 无法直观表达关节速度与末端速度的线性映射关 系。雅可比矩阵分为速度雅可比矩阵和角速度雅可 比矩阵, 速度雅可比矩阵可通过封闭矢量法求解, 相对构造法简化了求解过程, 但是角速度雅可比矩 阵一直没有合适的求解方法。XI 等 ${ }^{[10]}$ 改进了矢量积 法, 将基坐标系建在中间关节上, 减少了构造过程 计算量, 但尚未克服构造法的缺点。为此, 本文提 出矢量解析法, 考虑机械臂关节速度与末端速度的 关系, 将关节速度矢量在基坐标系下分解, 分别在 $X 、 Y$ 和 $Z$ 轴上合成末端角速度分量, 直接建立关 节速度与末端角速度的联系。相比构造法的抽象描 述和大量计算, 此方法化抽象为具象, 概念清晰、 形象直观, 大大简化计算。

\section{1 双臂协调机器人相对动力学建模}

\section{1 矢量解析法}

在机械臂动力学建模中, 角速度雅可比矩阵建 立了机械臂关节速度与末端角速度之间联系, 其运
用矢量解析法求解模型如图 1 所示。首先将每个关 节速度矢量分别向基坐标系 $X 、 Y$ 和 $Z$ 轴投影, 即 将 $\dot{\boldsymbol{q}}_{i}$ 分解为 $\dot{q}_{i x} 、 \dot{q}_{i y} 、 \dot{q}_{i z}(i=1,2,3)$, 再将各坐标轴 上投影合成末端角速度分量 $\omega_{x} 、 \omega_{y} 、 \omega_{z}$, 整合写 成矩阵形式, 求得角速度雅可比矩阵表达式

$$
\left\{\begin{array}{l}
\omega_{x}=\dot{q}_{1 x}+\dot{q}_{2 x}+\dot{q}_{3 x} \\
\omega_{y}=\dot{q}_{1 y}+\dot{q}_{2 y}+\dot{q}_{3 y} \\
\omega_{z}=\dot{q}_{1 z}+\dot{q}_{2 z}+\dot{q}_{3 z}
\end{array}\right.
$$

因 $\dot{\boldsymbol{q}}_{1}$ 始终与基坐标系 $Z$ 轴同向, 所以 $\dot{q}_{1 x}=\dot{q}_{1 y}=0$, $\dot{q}_{1 z}=\dot{q}_{1} \circ \dot{\boldsymbol{q}}_{2}$ 和 $\dot{\boldsymbol{q}}_{3}$ 始终与基坐标系 $Z$ 轴垂直且方向与 关节 1 转动角度 $q_{1}$ 有关, 所以 $\dot{q}_{2 x}=\dot{q}_{2} \sin q_{1}$, $\dot{q}_{2 y}=\dot{q}_{2} \cos q_{1}, \quad \dot{q}_{3 x}=\dot{q}_{3} \sin q_{1}, \quad \dot{q}_{3 y}=\dot{q}_{3} \cos q_{1}$, $\dot{q}_{2 z}=\dot{q}_{3 z}=0$ 。代入式(1)得

$$
\left\{\begin{array}{l}
\omega_{x}=\left(\dot{q}_{2}+\dot{q}_{3}\right) \sin q_{1} \\
\omega_{y}=-\left(\dot{q}_{2}+\dot{q}_{3}\right) \cos q_{1} \\
\omega_{z}=\dot{q}_{1}
\end{array}\right.
$$

用矩阵形式表示式(2)

$$
\left[\begin{array}{c}
\omega_{x} \\
\omega_{y} \\
\omega_{z}
\end{array}\right]=\left[\begin{array}{ccc}
0 & \sin q_{1} & \sin q_{1} \\
0 & -\cos q_{1} & -\cos q_{1} \\
1 & 0 & 0
\end{array}\right]_{J_{\omega}}\left[\begin{array}{l}
\dot{q}_{1} \\
\dot{q}_{2} \\
\dot{q}_{3}
\end{array}\right]
$$

即 $\boldsymbol{\omega}=\boldsymbol{J}_{\omega} \dot{\boldsymbol{q}}, \boldsymbol{\omega}=\left[\begin{array}{lll}\omega_{x} & \omega_{y} & \omega_{z}\end{array}\right]^{\mathrm{T}}$ 为机械臂末端角速 度, $\omega_{x} 、 \omega_{y} 、 \omega_{z}$ 分别为机械臂末端在基坐标系中 绕 $X 、 Y 、 Z$ 轴旋转的角速度； $\boldsymbol{J}_{\omega}$ 为所求的角速度 雅可比矩阵; $\dot{\boldsymbol{q}}=\left[\begin{array}{lll}\dot{q}_{1} & \dot{q}_{2} & \dot{q}_{3}\end{array}\right]^{\mathrm{T}}$ 为机械臂关节速度。

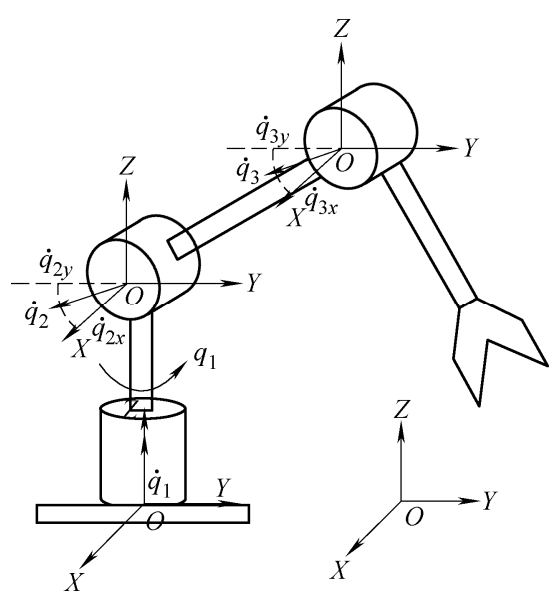

图 1 角速度雅可比矩阵求解模型

利用构造法求解角速度雅可比矩阵是复杂繁琐 的, 以微分变换法为例, 假设机械臂有 $n$ 个自由度, 首先需计算各连杆变换 ${ }^{0} \boldsymbol{T}_{1},{ }^{1} \boldsymbol{T}_{2}, \cdots,{ }^{n-1} \boldsymbol{T}_{n}$, 其通式为

$$
\boldsymbol{T}=\left[\begin{array}{cccc}
n_{x} & o_{x} & a_{x} & p_{x} \\
n_{y} & o_{y} & a_{y} & p_{y} \\
n_{z} & o_{z} & a_{z} & p_{z} \\
0 & 0 & 0 & 1
\end{array}\right]
$$


然后建立各连杆至木端连杆变换 ${ }^{0} \boldsymbol{T}_{n},{ }^{1} \boldsymbol{T}_{n}, \cdots,{ }^{n-1} \boldsymbol{T}_{n}$ 。 因为各连杆变换都是 4 阶方阵, 随着机械臂自由度 增多, 各连杆至末端连杆变换求解计算量大, 如 ${ }^{0} \boldsymbol{T}_{n}={ }^{0} \boldsymbol{T}_{1}{ }^{1} \boldsymbol{T}_{2} \cdots{ }^{n-1} \boldsymbol{T}_{n}, n$ 个 4 阶方阵相乘计算复杂且 容易出错。最后计算角速度雅可比矩阵各项, 如图 1 模型, $\boldsymbol{J}_{\omega}=\left[\begin{array}{lll}\boldsymbol{J}_{\omega 1} & \boldsymbol{J}_{\omega 2} & \boldsymbol{J}_{\omega 3}\end{array}\right]^{\mathrm{T}}, \boldsymbol{J}_{\omega 1}=\left[\begin{array}{lll}n_{z} & o_{z} & a_{z}\end{array}\right]^{\mathrm{T}}$, 其中 $n_{z}, O_{z}, a_{z}$ 是 $^{0} \boldsymbol{T}_{3}$ 中的元素, $\boldsymbol{J}_{\omega 2}$ 对应 ${ }^{1} \boldsymbol{T}_{3}, \boldsymbol{J}_{\omega 3}$ 对应 ${ }^{2} \boldsymbol{T}_{3}$ 。则利用微分变换法求解结果

$$
\begin{gathered}
\boldsymbol{\omega}=\left[\begin{array}{lcl}
\boldsymbol{J}_{\omega 1} & \boldsymbol{J}_{\omega 2} & \boldsymbol{J}_{\omega 3}
\end{array}\right] \dot{\boldsymbol{q}}= \\
{\left[\begin{array}{ccc}
0 & \sin q_{1} & \sin q_{1} \\
0 & -\cos q_{1} & -\cos q_{1} \\
1 & 0 & 0
\end{array}\right]_{J_{\omega}}\left[\begin{array}{l}
\dot{q}_{1} \\
\dot{q}_{2} \\
\dot{q}_{3}
\end{array}\right]}
\end{gathered}
$$

对比矢量解析法与微分变换法, 可见微分变换 法是基于机械臂运动学连杆变换构造角速度雅可比 矩阵, 不仅理论推导过程抽象难懂, 而且计算过程 繁杂, 不如矢量解析法清晰形象。另外通过计算, 上述两方法得出的结果相同, 验证了矢量解析法的 正确性。

\section{2 相对雅可比矩阵}

相对雅可比矩阵 ${ }^{[11-12]}$ 区别于单臂雅可比矩阵, 是建立两机械臂关节速度与末端相对速度之间联 系, 将两臂整合成一个系统, 视为单机械臂处理。 为避免推导相对雅可比矩阵全新形式的复杂计算, 利用两机械臂各自参数, 对单机械臂雅可比矩阵进 行必要转换得到相对雅可比矩阵的简洁表达 ${ }^{[13]}$ 。

首先, 建立空间六自由度双臂机器人结构 图, 如图 2 所示。在 $A B$ 连线中点建立空间运 动惯性坐标系 $O X Y Z$, 根据后置法建立各连杆 相对坐标系 $O_{i} X_{i} Y_{i} Z_{i}(i=1,2, \cdots, 6)$, 各连杆长度为 $l_{i}(i=1,2, \cdots, 6), A O$ 和 $B O$ 长度为 $l$, 各关节质量为

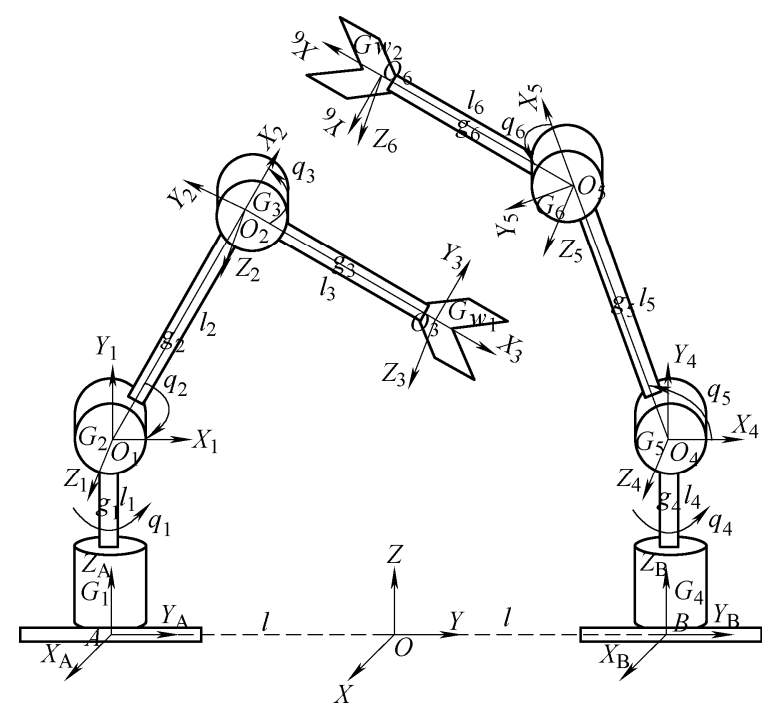

图 2 空间 6 自由度双臂机器人结构图
$G_{i}(i=1,2, \cdots, 6)$, 各机械臂末端执行器和夹持物体总 体质量为 $G_{W 1} 、 G_{W 2}$, 各连杆质量为 $g_{i}(i=1,2, \cdots, 6)$ 。

根据建立的双臂模型, 分析得到连杆 6 坐标系 相对 $A$ 坐标系旋转矩阵变换关系与 $O_{6}$ 到 $A$ 点坐标 旋转和平移复合变换关系

$$
\begin{gathered}
{ }^{A} \boldsymbol{R}_{O_{3}}{ }^{{ }_{3}} \boldsymbol{R}_{O_{6}}={ }^{A} \boldsymbol{R}_{B}{ }^{B} \boldsymbol{R}_{O_{6}} \\
{ }^{A} \boldsymbol{p}_{O_{3}}+{ }^{A} \boldsymbol{R}_{O_{3}}{ }^{O_{3}} \boldsymbol{p}_{O_{6}}={ }^{A} \boldsymbol{p}_{B}+{ }^{A} \boldsymbol{R}_{B}{ }^{B} \boldsymbol{p}_{O_{6}}
\end{gathered}
$$

对式(5)、(6)变换求导可得两机械臂末端相对速度和 角速度与关节速度之间关系

$$
\begin{gathered}
{\left[\begin{array}{c}
\dot{\boldsymbol{p}}_{R} \\
\boldsymbol{\omega}_{R}
\end{array}\right]=} \\
{\left[\begin{array}{c}
-{ }^{O_{3}} \boldsymbol{R}_{A} \boldsymbol{J}_{P A} \dot{\boldsymbol{q}}_{A}+\boldsymbol{S}\left(\boldsymbol{p}_{R}\right)^{O_{3}} \boldsymbol{R}_{A} \boldsymbol{J}_{\omega A} \dot{\boldsymbol{q}}_{A}+{ }^{O_{3}} \boldsymbol{R}_{B} \boldsymbol{J}_{P B} \dot{\boldsymbol{q}}_{B} \\
-{ }^{O_{3}} \boldsymbol{R}_{A} \boldsymbol{J}_{\omega A} \dot{\boldsymbol{q}}_{A}+{ }^{O_{3}} \boldsymbol{R}_{B} \boldsymbol{J}_{\omega B} \dot{\boldsymbol{q}}_{B}
\end{array}\right]=} \\
{\left[\begin{array}{ccc}
\boldsymbol{I} & -\boldsymbol{S}\left(\boldsymbol{p}_{R}\right) \\
\boldsymbol{0} & \boldsymbol{I}
\end{array}\right]\left[\begin{array}{cc}
-{ }^{O_{3}} \boldsymbol{R}_{A} & \boldsymbol{0} \\
\boldsymbol{0} & -{ }^{O_{3}} \boldsymbol{R}_{A}
\end{array}\right]\left[\begin{array}{l}
\boldsymbol{J}_{P A} \\
\boldsymbol{J}_{\omega A}
\end{array}\right]} \\
\left.\left[\begin{array}{cc}
{ }^{O_{3}} \boldsymbol{R}_{B} & \boldsymbol{0} \\
\boldsymbol{0} & { }^{O_{3}} \boldsymbol{R}_{B}
\end{array}\right]\left[\begin{array}{l}
\boldsymbol{J}_{P B} \\
\boldsymbol{J}_{\omega B}
\end{array}\right]\right]\left[\begin{array}{l}
\dot{\boldsymbol{q}}_{A} \\
\dot{\boldsymbol{q}}_{B}
\end{array}\right]
\end{gathered}
$$

式中, $\dot{\boldsymbol{p}}_{R}=\left[\begin{array}{lll}\dot{x}_{R} & \dot{y}_{R} & \dot{z}_{R}\end{array}\right]^{\mathrm{T}}$ 为 $O_{6}$ 相对 $O_{3}$ 点速度, $\boldsymbol{\omega}_{R}=\left[\begin{array}{lll}\dot{\alpha}_{R} & \dot{\beta}_{R} & \dot{\gamma}_{R}\end{array}\right]^{\mathrm{T}}$ 为 $O_{6}$ 相对 $O_{3}$ 点角速度, $\boldsymbol{I}$ 为 3 阶单位阵, $\boldsymbol{S}\left(\boldsymbol{p}_{R}\right)^{[14]}$ 为输入矢量 $\boldsymbol{p}_{R}$ 的斜对称矩阵

$$
\boldsymbol{S}\left(\boldsymbol{p}_{R}\right)=\left[\begin{array}{ccc}
0 & -z_{R} & y_{R} \\
z_{R} & 0 & -x_{R} \\
-y_{R} & x_{R} & 0
\end{array}\right]
$$

$$
\begin{aligned}
x_{R}= & {\left[l_{5} \cos q_{5}+l_{6} \cos \left(q_{5}+q_{6}\right)\right] \cos q_{4}-} \\
& {\left[l_{2} \cos q_{2}+l_{3} \cos \left(q_{2}+q_{3}\right)\right] \cos q_{1} }
\end{aligned}
$$

$$
y_{R}=2 l+\left[l_{5} \cos q_{5}+l_{6} \cos \left(q_{5}+q_{6}\right)\right] \sin q_{4}-
$$

$\left[l_{2} \cos q_{2}+l_{3} \cos \left(q_{2}+q_{3}\right)\right] \sin q_{1}$

$$
\begin{aligned}
z_{R}= & {\left[l_{4}+l_{5} \sin q_{5}+l_{6} \sin \left(q_{5}+q_{6}\right)\right]-} \\
& {\left[l_{1}+l_{2} \sin q_{2}+l_{3} \sin \left(q_{2}+q_{3}\right)\right] }
\end{aligned}
$$

${ }^{O_{3}} \boldsymbol{R}_{A} 、{ }^{O_{3}} \boldsymbol{R}_{B}$ 分别为点 $A 、 B$ 坐标系相对连杆 3 坐标 系的旋转矩阵变换, ${ }^{O_{3}} \boldsymbol{R}_{A}={ }^{O_{3}} \boldsymbol{R}_{O_{2}}{ }^{O_{2}} \boldsymbol{R}_{O_{1}}{ }^{O_{1}} \boldsymbol{R}_{A}$, ${ }^{O_{3}} \boldsymbol{R}_{B}={ }^{O_{3}} \boldsymbol{R}_{O_{2}}{ }^{O_{2}} \boldsymbol{R}_{O_{1}}{ }^{O_{1}} \boldsymbol{R}_{A}{ }^{A} \boldsymbol{R}_{B},{ }^{i} \boldsymbol{R}_{j}$ 为 $j$ 坐标系相对 $i$ 坐 标系的旋转矩阵变换。因为 $B$ 坐标系相对 $A$ 坐标系 没有旋转变换, 所以 ${ }^{A} \boldsymbol{R}_{B}=\boldsymbol{I},{ }^{{ }_{3}} \boldsymbol{R}_{A}={ }^{O_{3}} \boldsymbol{R}_{B}$ 。

$$
\begin{aligned}
{ }^{O_{3}} \boldsymbol{R}_{O_{2}} & =\left[\begin{array}{ccc}
\cos q_{3} & -\sin q_{3} & 0 \\
\sin q_{3} & \cos q_{3} & 0 \\
0 & 0 & 1
\end{array}\right]^{\mathrm{T}} \\
{ }^{O_{2}} \boldsymbol{R}_{O_{1}} & =\left[\begin{array}{ccc}
\cos q_{2} & -\sin q_{2} & 0 \\
\sin q_{2} & \cos q_{2} & 0 \\
0 & 0 & 1
\end{array}\right]^{\mathrm{T}}
\end{aligned}
$$




$$
{ }^{O_{1}} \boldsymbol{R}_{A}=\left[\begin{array}{ccc}
1 & 0 & 0 \\
0 & 0 & -1 \\
0 & 1 & 0
\end{array}\right]^{\mathrm{T}}\left[\begin{array}{ccc}
\cos q_{1} & -\sin q_{1} & 0 \\
\sin q_{1} & \cos q_{1} & 0 \\
0 & 0 & 1
\end{array}\right]^{\mathrm{T}}
$$

$\boldsymbol{J}_{A}=\left[\begin{array}{ll}\boldsymbol{J}_{P A} & \boldsymbol{J}_{\omega A}\end{array}\right]^{\mathrm{T}} 、 \boldsymbol{J}_{B}=\left[\begin{array}{ll}\boldsymbol{J}_{P B} & \boldsymbol{J}_{\omega B}\end{array}\right]^{\mathrm{T}}$ 分别为两机械 臂独立雅可比矩阵。

$$
\begin{aligned}
& \boldsymbol{J}_{A}=\left[\begin{array}{ccc}
J_{A 11} & J_{A 12} & J_{A 13} \\
J_{A 21} & J_{A 22} & J_{A 23} \\
J_{A 31} & J_{A 32} & J_{A 33} \\
0 & \sin q_{1} & \sin q_{1} \\
0 & -\cos q_{1} & -\cos q_{1} \\
1 & 0 & 0
\end{array}\right] \\
& J_{A 11}=-\left[l_{2} \cos q_{2}+l_{3} \cos \left(q_{2}+q_{3}\right)\right] \sin q_{1} \\
& J_{A 12}=-\left[l_{2} \sin q_{2}+l_{3} \sin \left(q_{2}+q_{3}\right)\right] \cos q_{1} \\
& J_{A 13}=-l_{3} \sin \left(q_{2}+q_{3}\right) \cos q_{1} \\
& J_{A 21}=\left[l_{2} \cos q_{2}+l_{3} \cos \left(q_{2}+q_{3}\right)\right] \cos q_{1} \\
& J_{\text {A22 }}=-\left[l_{2} \sin q_{2}+l_{3} \sin \left(q_{2}+q_{3}\right)\right] \sin q_{1} \\
& J_{\text {A23 }}=-l_{3} \sin \left(q_{2}+q_{3}\right) \sin q_{1} \\
& J_{\text {A31 }}=0 \\
& J_{\text {A32 }}=l_{2} \cos q_{2}+l_{3} \cos \left(q_{2}+q_{3}\right) \\
& J_{A 33}=l_{3} \cos \left(q_{2}+q_{3}\right) \\
& \boldsymbol{J}_{B}=\left[\begin{array}{ccc}
J_{B 11} & J_{B 12} & J_{B 13} \\
J_{B 21} & J_{B 22} & J_{B 23} \\
J_{B 31} & J_{B 32} & J_{B 33} \\
0 & \sin q_{4} & \sin q_{4} \\
0 & -\cos q_{4} & -\cos q_{4} \\
1 & 0 & 0
\end{array}\right] \\
& J_{B 11}=-\left[l_{5} \cos q_{5}+l_{6} \cos \left(q_{5}+q_{6}\right)\right] \sin q_{4} \\
& J_{B 12}=-\left[l_{5} \sin q_{5}+l_{6} \sin \left(q_{5}+q_{6}\right)\right] \cos q_{4} \\
& J_{B 13}=-l_{6} \sin \left(q_{5}+q_{6}\right) \cos q_{4} \\
& J_{B 21}=\left[l_{5} \cos q_{5}+l_{6} \cos \left(q_{5}+q_{6}\right)\right] \cos q_{4} \\
& J_{\text {B22 }}=-\left[l_{5} \sin q_{5}+l_{6} \sin \left(q_{5}+q_{6}\right)\right] \sin q_{4} \\
& J_{B 23}=-l_{6} \sin \left(q_{5}+q_{6}\right) \sin q_{4} \\
& J_{B 31}=0 \\
& J_{B 32}=l_{5} \cos q_{5}+l_{6} \cos \left(q_{5}+q_{6}\right) \\
& J_{B 33}=l_{6} \cos \left(q_{5}+q_{6}\right)
\end{aligned}
$$

相对雅可比矩阵为

$$
\begin{gathered}
\boldsymbol{J}_{R}=\left[\begin{array}{lll}
-\boldsymbol{\Psi}_{R}{ }^{O_{3}} \boldsymbol{\Omega}_{A} \boldsymbol{J}_{A} & { }^{O_{3}} \boldsymbol{\Omega}_{B} \boldsymbol{J}_{B}
\end{array}\right] \\
\boldsymbol{\psi}_{R}=\left[\begin{array}{cc}
\boldsymbol{I} & -\boldsymbol{S}\left(\boldsymbol{p}_{R}\right) \\
\boldsymbol{0} & \boldsymbol{I}
\end{array}\right] \quad{ }^{j} \boldsymbol{\Omega}_{i}=\left[\begin{array}{cc}
{ }^{j} \boldsymbol{R}_{i} & \boldsymbol{0} \\
\boldsymbol{0} & { }^{j} \boldsymbol{R}_{i}
\end{array}\right]
\end{gathered}
$$

相对雅可比矩阵建立两机械臂之间联系，描述 两臂末端相对关系, 为下面相对动力学建模提供理 论支持。

\section{3 相对动力学建模}

相对动力学模型对应相对雅可比矩阵, 是利用 相对雅可比矩阵对一般动力学模型的进一步推导。 根据图 2 所示模型, 利用拉格朗日方程

$$
\frac{\mathrm{d}}{\mathrm{d} t}\left(\frac{\partial L}{\partial \dot{q}_{i}}\right)-\frac{\partial L}{\partial q_{i}}=\tau_{i}
$$

式中, $L=K-P, K$ 为系统动能, $P$ 为系统势能, $\boldsymbol{q}=\left[q_{1} q_{2} \cdots q_{i}\right]^{\mathrm{T}}$ 为系统广义坐标。

式(9)经过简化, 得双臂动力学模型为

$$
\boldsymbol{M}(\boldsymbol{q}) \ddot{\boldsymbol{q}}+\boldsymbol{H}(\boldsymbol{q}, \dot{q})+\boldsymbol{G}(\boldsymbol{q})=\boldsymbol{\tau}
$$

式中, $M(q)$ 为对称、正定的质量矩阵

$$
\boldsymbol{M}(\boldsymbol{q})=\left[\begin{array}{ccc}
M_{11} & \cdots & M_{16} \\
\vdots & & \vdots \\
M_{61} & \cdots & M_{66}
\end{array}\right]
$$

$$
M_{11}=\left(G_{3}+\frac{1}{3} g_{2}\right) l_{2}^{2} \cos ^{2} q_{2}+
$$$$
G_{W 1}\left[l_{3} \cos \left(q_{2}+q_{3}\right)+l_{2} \cos q_{2}\right]^{2}+
$$$$
g_{3}\left[\frac{1}{3} l_{3}^{2} \cos ^{2}\left(q_{2}+q_{3}\right)+l_{2}^{2} \cos ^{2} q_{2}\right]
$$

$$
\begin{aligned}
M_{22}= & G_{3} l_{2}^{2}+\frac{1}{3} g_{2} l_{2}^{2}+G_{W 1}\left(l_{2}^{2}+l_{3}^{2}+2 l_{2} l_{3} \cos q_{3}\right)+ \\
& g_{3}\left(l_{2}^{2}+\frac{1}{3} l_{3}^{2}+l_{2} l_{3} \cos q_{3}\right)
\end{aligned}
$$

$M_{23}=G_{W 1}\left(l_{3}^{2}+l_{2} l_{3} \cos q_{3}\right)+g_{3}\left(\frac{1}{3} l_{3}^{2}+\frac{1}{2} l_{2} l_{3} \cos q_{3}\right)$

$$
M_{32}=G_{W 1}\left(l_{3}^{2}+l_{2} l_{3} \cos q_{3}\right)+g_{3}\left(\frac{1}{3} l_{3}^{2}+\frac{1}{2} l_{2} l_{3} \cos q_{3}\right)
$$

$$
M_{33}=G_{W 1} l_{3}^{2}+\frac{1}{3} g_{3} l_{3}^{2}
$$$$
M_{44}=\left(G_{6}+\frac{1}{3} g_{5}\right) l_{5}^{2} \cos ^{2} q_{5}+
$$$$
G_{W 2}\left[l_{6} \cos \left(q_{5}+q_{6}\right)+l_{5} \cos q_{5}\right]^{2}+
$$

$g_{6}\left[\frac{1}{3} l_{6}^{2} \cos ^{2}\left(q_{5}+q_{6}\right)+l_{5}^{2} \cos ^{2} q_{5}\right]$

$$
M_{55}=G_{6} l_{5}^{2}+\frac{1}{3} g_{5} l_{5}^{2}+G_{W 2}\left(l_{5}^{2}+l_{6}^{2}+2 l_{5} l_{6} \cos q_{6}\right)+
$$

$$
g_{6}\left(l_{5}^{2}+\frac{1}{3} l_{6}^{2}+l_{5} l_{6} \cos q_{6}\right)
$$

$$
\begin{aligned}
& M_{56}=G_{W 2}\left(l_{6}^{2}+l_{5} l_{6} \cos q_{6}\right)+g_{6}\left(\frac{1}{3} l_{6}^{2}+\frac{1}{2} l_{5} l_{6} \cos q_{6}\right) \\
& M_{65}=G_{W 2}\left(l_{6}^{2}+l_{5} l_{6} \cos q_{6}\right)+g_{6}\left(\frac{1}{3} l_{6}^{2}+\frac{1}{2} l_{5} l_{6} \cos q_{6}\right)
\end{aligned}
$$




$$
M_{66}=G_{W 2} l_{6}^{2}+\frac{1}{3} g_{6} l_{6}^{2}
$$

$\boldsymbol{H}(\boldsymbol{q}, \dot{\boldsymbol{q}})$ 为包括哥氏力、离心力的广义力矢量

$$
\begin{gathered}
\boldsymbol{H}(\boldsymbol{q}, \dot{\boldsymbol{q}})=\left[\begin{array}{ccc}
H_{11} & \cdots & H_{16} \\
\vdots & & \vdots \\
H_{61} & \cdots & H_{66}
\end{array}\right]\left[\begin{array}{c}
\dot{q}_{1}^{2} \\
\vdots \\
\dot{q}_{6}^{2}
\end{array}\right]+ \\
{\left[\begin{array}{ccc}
h_{11} & \cdots & h_{16} \\
\vdots & & \vdots \\
h_{61} & \cdots & h_{66}
\end{array}\right]\left[\begin{array}{c}
\dot{q}_{1} \dot{q}_{2} \\
\dot{q}_{1} \dot{q}_{3} \\
\dot{q}_{2} \dot{q}_{3} \\
\dot{q}_{4} \dot{q}_{5} \\
\dot{q}_{4} \dot{q}_{6} \\
\dot{q}_{5} \dot{q}_{6}
\end{array}\right]}
\end{gathered}
$$

$H_{21}=\left(G_{3}+\frac{1}{3} g_{2}\right) l_{2}^{2} \cos q_{2} \sin q_{2}+$

$G_{W 1}\left[l_{3} \cos \left(q_{2}+q_{3}\right)+l_{2} \cos q_{2}\right] \times$

$\left[l_{3} \sin \left(q_{2}+q_{3}\right)+l_{2} \sin q_{2}\right]+$

$g_{3}\left[\frac{1}{3} l_{3}^{2} \cos \left(q_{2}+q_{3}\right) \sin \left(q_{2}+q_{3}\right)+l_{2}^{2} \cos q_{2} \sin q_{2}\right]$

$$
H_{23}=-G_{W 1} l_{2} l_{3} \sin q_{3}-\frac{1}{2} g_{3} l_{2} l_{3} \sin q_{3}
$$

$H_{31}=G_{W 1} l_{3} \sin \left(q_{2}+q_{3}\right)\left[l_{3} \cos \left(q_{2}+q_{3}\right)+l_{2} \cos q_{2}\right]+$

$\frac{1}{3} g_{3} l_{3}^{2} \cos \left(q_{2}+q_{3}\right) \sin \left(q_{2}+q_{3}\right)$

$$
H_{32}=G_{W 1} l_{2} l_{3} \sin q_{3}+\frac{1}{2} g_{3} l_{2} l_{3} \sin q_{3}
$$

$H_{54}=\left(G_{6}+\frac{1}{3} g_{5}\right) l_{5}^{2} \cos q_{5} \sin q_{5}+$

$G_{W 2}\left[l_{6} \cos \left(q_{5}+q_{6}\right)+l_{5} \cos q_{5}\right] \times$

$\left[l_{6} \sin \left(q_{5}+q_{6}\right)+l_{5} \sin q_{5}\right]+$

$g_{6}\left[\frac{1}{3} l_{6}^{2} \cos \left(q_{5}+q_{6}\right) \sin \left(q_{5}+q_{6}\right)+l_{5}^{2} \cos q_{5} \sin q_{5}\right]$

$$
H_{56}=-G_{W 2} l_{5} l_{6} \sin q_{6}-\frac{1}{2} g_{6} l_{5} l_{6} \sin q_{6}
$$

$H_{64}=G_{W 2} l_{6} \sin \left(q_{5}+q_{6}\right)\left[l_{6} \cos \left(q_{5}+q_{6}\right)+l_{5} \cos q_{5}\right]+$

$\frac{1}{3} g_{6} l_{6}^{2} \cos \left(q_{5}+q_{6}\right) \sin \left(q_{5}+q_{6}\right)$

$$
H_{65}=G_{W 2} l_{5} l_{6} \sin q_{6}+\frac{1}{2} g_{6} l_{5} l_{6} \sin q_{6}
$$

$h_{11}=-\left(2 G_{3}+\frac{2}{3} g_{2}\right) l_{2}^{2} \cos q_{2} \sin q_{2}-$

$2 G_{W 1}\left[l_{3} \cos \left(q_{2}+q_{3}\right)+l_{2} \cos q_{2}\right] \times$

$\left[l_{3} \sin \left(q_{2}+q_{3}\right)+l_{2} \sin q_{2}\right]-$

$g_{3}\left[\frac{2}{3} l_{3}^{2} \cos \left(q_{2}+q_{3}\right) \sin \left(q_{2}+q_{3}\right)+2 l_{2}^{2} \cos q_{2} \sin q_{2}\right]$

$h_{12}=-2 G_{W 1} l_{3} \sin \left(q_{2}+q_{2}\right)\left[l_{3} \cos \left(q_{2}+q_{3}\right)+l_{2} \cos q_{2}\right]-$

$\frac{2}{3} g_{3} l_{3}^{2} \cos \left(q_{2}+q_{3}\right) \sin \left(q_{2}+q_{3}\right)$ $h_{23}=-2 G_{W 1} l_{2} l_{3} \sin q_{3}-g_{3} l_{2} l_{3} \sin q_{3}$

$h_{44}=-\left(2 G_{6}+\frac{2}{3} g_{5}\right) l_{5}^{2} \cos q_{5} \sin q_{5}-$

$2 G_{W 2}\left[l_{6} \cos \left(q_{5}+q_{6}\right)+l_{5} \cos q_{5}\right] \times$

$\left[l_{6} \sin \left(q_{5}+q_{6}\right)+l_{5} \sin q_{5}\right]-$

$g_{6}\left[\frac{2}{3} l_{6}^{2} \cos \left(q_{5}+q_{6}\right) \sin \left(q_{5}+q_{6}\right)+2 l_{5}^{2} \cos q_{5} \sin q_{5}\right]$

$h_{45}=-2 G_{W 5} l_{6} \sin \left(q_{5}+q_{5}\right)\left[l_{6} \cos \left(q_{5}+q_{6}\right)+l_{5} \cos q_{5}\right]-$

$\frac{2}{3} g_{6} l_{6}^{2} \cos \left(q_{5}+q_{6}\right) \sin \left(q_{5}+q_{6}\right)$

$h_{56}=-2 G_{W 2} l_{5} l_{6} \sin q_{6}-g_{6} l_{5} l_{6} \sin q_{6}$

$\boldsymbol{G}(\boldsymbol{q})=\left[\begin{array}{lll}P_{1} & \cdots & P_{6}\end{array}\right]^{\mathrm{T}}$ 为重力项

$P_{2}=G_{3} l_{2} g \cos q_{2}+\frac{1}{2} g_{2} l_{2} g \cos q_{2}+$

$G_{W 1} g\left[l_{3} \cos \left(q_{2}+q_{3}\right)+l_{2} \cos q_{2}\right]+$

$g_{3} g\left[\frac{1}{2} l_{3} \cos \left(q_{2}+q_{3}\right)+l_{2} \cos q_{2}\right]$

$P_{3}=G_{W 1} g l_{3} \cos \left(q_{2}+q_{3}\right)+\frac{1}{2} g_{3} g l_{3} \cos \left(q_{2}+q_{3}\right)$

$P_{5}=G_{6} l_{5} g \cos q_{5}+\frac{1}{2} g_{5} l_{5} g \cos q_{5}+$

$G_{W 2} g\left[l_{6} \cos \left(q_{5}+q_{6}\right)+l_{5} \cos q_{5}\right]+$

$g_{6} g\left[\frac{1}{2} l_{6} \cos \left(q_{5}+q_{6}\right)+l_{5} \cos q_{5}\right]$

$P_{6}=G_{W 2} g l_{6} \cos \left(q_{5}+q_{6}\right)+\frac{1}{2} g_{6} g l_{6} \cos \left(q_{5}+q_{6}\right)$

式中, $M(q) 、 H(q, \dot{q})$ 和 $\boldsymbol{G}(\boldsymbol{q})$ 中未写出的元素具体 表达为零, $\boldsymbol{\tau}=\left[\begin{array}{llllll}\tau_{1} & \tau_{2} & \tau_{3} & \tau_{4} & \tau_{5} & \tau_{6}\end{array}\right]^{\mathrm{T}}$ 为关节力 矩, $\boldsymbol{q}=\left[\begin{array}{llllll}q_{1} & q_{2} & q_{3} & q_{4} & q_{5} & q_{6}\end{array}\right]^{\mathrm{T}}$ 为广义坐标, 即 各关节速度。

利用相对雅可比矩阵得

$$
\dot{\boldsymbol{X}}_{R}=\boldsymbol{J}_{R} \dot{\boldsymbol{q}}
$$

$\dot{\boldsymbol{X}}_{R}=\left[\begin{array}{ll}\dot{\boldsymbol{p}}_{R} & \boldsymbol{\omega}_{R}\end{array}\right]^{\mathrm{T}}=\left[\begin{array}{llllll}\dot{x}_{R} & \dot{y}_{R} & \dot{\bar{z}}_{R} & \dot{\alpha}_{R} & \dot{\beta}_{R} & \dot{\gamma}_{R}\end{array}\right]^{\mathrm{T}}$ 为两 机械臂末端相对速度和角速度, 相对雅可比矩阵 $\boldsymbol{J}_{R}$ 如式(8)所示, 式(11)微分得

$$
\delta \boldsymbol{X}_{R}=\boldsymbol{J}_{R} \delta \boldsymbol{q}
$$

$\delta \boldsymbol{q}$ 为关节虚位移, $\delta \boldsymbol{X}_{R}$ 为两机械臂末端相对虚位移 和虚角位移, 根据虚位移原理, 各关节所做虚功之 和与机械臂末端所做虚功相等

$$
\boldsymbol{\tau}^{\mathrm{T}} \delta \boldsymbol{q}=\boldsymbol{F}_{R}^{\mathrm{T}} \delta \boldsymbol{X}_{R}
$$

$\boldsymbol{F}_{R}=\left[\begin{array}{llllll}F_{R X} & F_{R Y} & F_{R Z} & \tau_{R X} & \tau_{R Y} & \tau_{R Z}\end{array}\right]^{\mathrm{T}}$ 为两机械臂 末端相对力和力矩, 将式(12)代入式(13)得

$$
\boldsymbol{\tau}^{\mathrm{T}} \delta \boldsymbol{q}=\boldsymbol{F}_{R}{ }^{\mathrm{T}} \boldsymbol{J}_{R} \delta \boldsymbol{q}
$$

化简得 


$$
\boldsymbol{\tau}=\boldsymbol{J}_{R}{ }^{\mathrm{T}} \boldsymbol{F}_{R}
$$

将式(15)代入式(10)得

$$
\boldsymbol{M}(\boldsymbol{q}) \ddot{\boldsymbol{q}}+\boldsymbol{H}(\boldsymbol{q}, \dot{\boldsymbol{q}})+\boldsymbol{G}(\boldsymbol{q})=\boldsymbol{\tau}=\boldsymbol{J}_{R}{ }^{\mathrm{T}} \boldsymbol{F}_{R}
$$

则两机械臂末端相对力, 即相对动力学模型为

$$
\begin{gathered}
\boldsymbol{F}_{R}=\left(\boldsymbol{J}_{R}^{\mathrm{T}}\right)^{+}[\boldsymbol{M}(\boldsymbol{q}) \ddot{\boldsymbol{q}}+\boldsymbol{H}(\boldsymbol{q}, \dot{\boldsymbol{q}})+\boldsymbol{G}(\boldsymbol{q})]+ \\
{\left[\boldsymbol{I}-\left(\boldsymbol{J}_{R}^{\mathrm{T}}\right)^{+} \boldsymbol{J}_{R}^{\mathrm{T}}\right] \boldsymbol{\delta}}
\end{gathered}
$$

式中, $\left(\boldsymbol{J}_{R}^{\mathrm{T}}\right)^{+}$为 $\boldsymbol{J}_{R}^{\mathrm{T}}$ 的广义逆矩阵, $\boldsymbol{\delta}$ 定义在 $\boldsymbol{J}_{R}^{\mathrm{T}}$ 的零 空间内, 即 $\boldsymbol{J}_{R}^{\mathrm{T}} \boldsymbol{\delta}=0$, 则

$$
\boldsymbol{F}_{R}=\left(\boldsymbol{J}_{R}^{\mathrm{T}}\right)^{+}[\boldsymbol{M}(\boldsymbol{q}) \ddot{\boldsymbol{q}}+\boldsymbol{H}(\boldsymbol{q}, \dot{\boldsymbol{q}})+\boldsymbol{G}(\boldsymbol{q})]+\boldsymbol{\delta}
$$

\section{2 仿真与试验}

经上述推导得到的式(18)建立了两机械臂末端 相对力与各关节参数之间联系, 下面的仿真与试验 将验证相对动力学模型的正确性。

\section{1 仿真条件设定}

根据试验对象, 安川双臂机器人 SDA10F, 在 Matlab2014 软件环境中进行仿真, 因试验没有涉及 零空间控制, 所以相对力表达式中 $\boldsymbol{\delta}$ 和 $\boldsymbol{\zeta}$ 设置为零。 为更好的模拟两机械臂关节实际运动情况, 设计了 关节运动速度和加速度, 使关节运动分为加速段、 匀速段和减速段。两机械臂各关节运动情况如图 $3 \sim 8$ 所示。

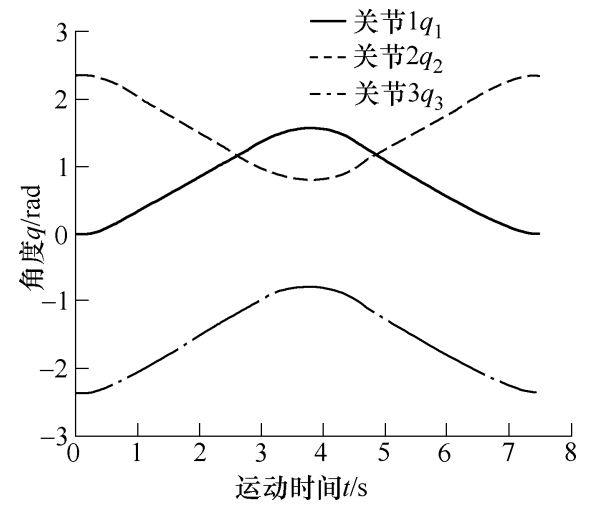

图 3 左机械臂关节转动角度

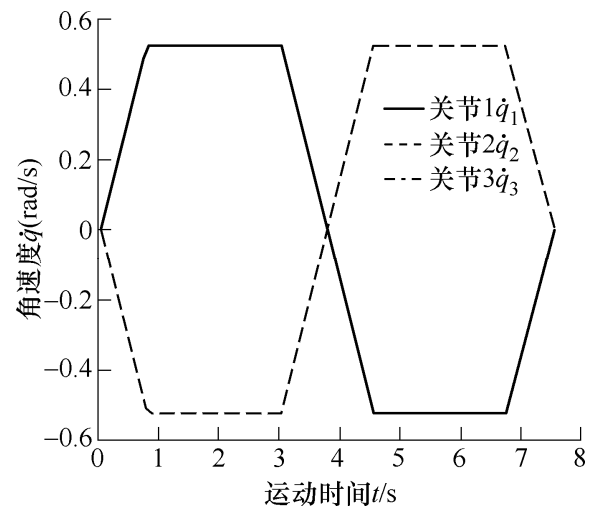

图 4 左机械臂关节转动速度

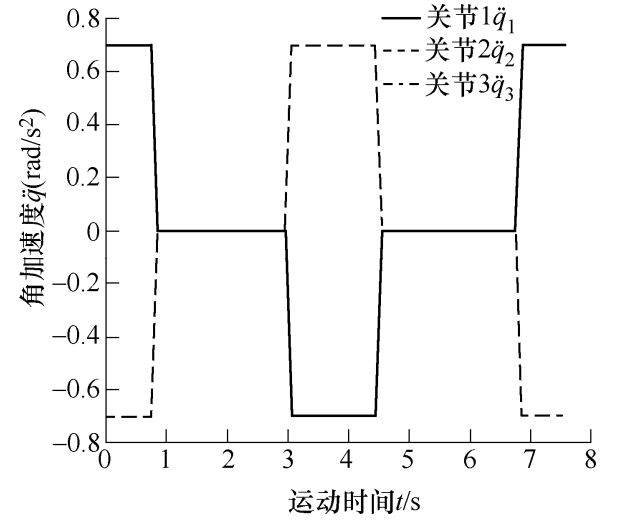

图 5 左机械臂关节转动加速度

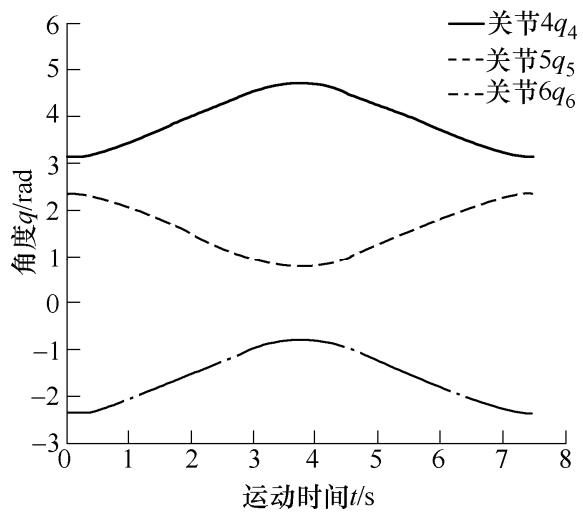

图 6 右机械臂关节转动角度

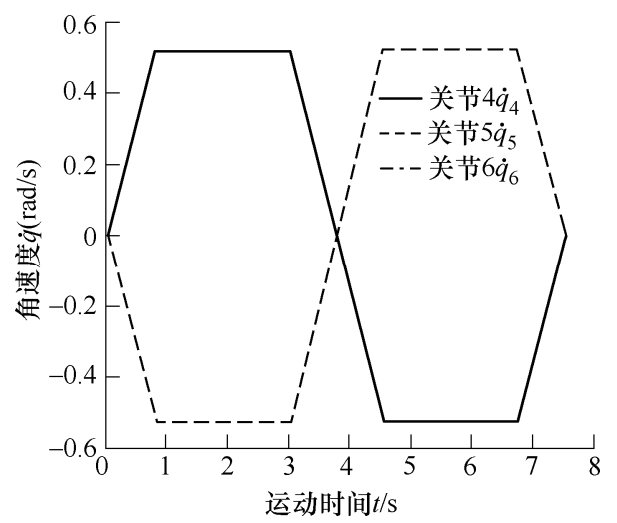

图 7 右机械臂关节转动速度

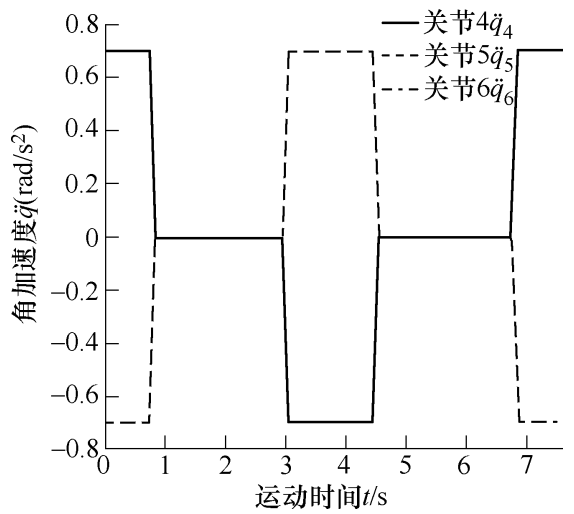

图 8 右机械臂关节转动加速度

安川双臂机器人 SDA10F 每个机械臂都有 7 自 由度, 根据图 2 双臂机器人结构, 试验时每个臂只 利用 3 个自由度，即第 $3 、 4 、 6$ 自由度，如图 9 所 
示。安川双臂机器人 SDA10F 没有具体单关节参数, 参考安川单臂机器人 SIA10F, 本体质量 $60 \mathrm{~kg}$, 垂 直和水平伸长度分别为 $1203 \mathrm{~mm} 、 720 \mathrm{~mm}$, 可认 为 SDA10F 由两个 SIA $10 \mathrm{~F}$ 和底座构成。参考上述 数据, 估计图 2 中双臂机器人参数, 各关节质量 $G_{1}=G_{4}=4 \mathrm{~kg}, G_{2}=G_{5}=3 \mathrm{~kg}, G_{3}=G_{6}=3 \mathrm{~kg}$ 。由 于利用的 3 个自由度是不连续的, 为了求得较准确 的仿真数据, 连杆 $2 、 3 、 5 、 6$ 参数包括本体关节, 与连杆 $1 、 2$ 不同。各连杆质量分别是 $g_{1}=g_{4}=1 \mathrm{~kg}$, $g_{2}=g_{5}=4 \mathrm{~kg}, g_{3}=g_{6}=4 \mathrm{~kg}$, 长度分别为 $l_{1}=l_{4}=30 \mathrm{~cm}, l_{2}=l_{5}=50 \mathrm{~cm}, l_{3}=l_{6}=20 \mathrm{~cm}$, 两 机械臂基座间距 $2 l=530 \mathrm{~mm}$, 由于没有安装机械臂 末端执行器, $G_{W 1} 、 G_{W 2}$ 忽略不计。

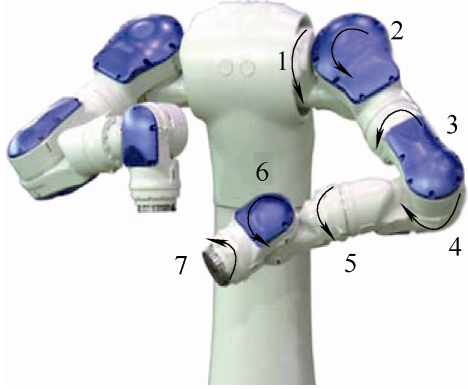

图 9 安川双臂机器人 SDA10F

\section{2 仿真与试验分析}

一个循环周期试验截图如图 10 所示, 运动过程 为从左到右, 从上到下的顺序。虽然各关节起始角 度位置不同, 但是两机械臂对应关节运动速度和加 速度相同。为方便试验和分析, 两机械臂运动状态 始终关于底座坚直轴线中心对称, 并循环运动。

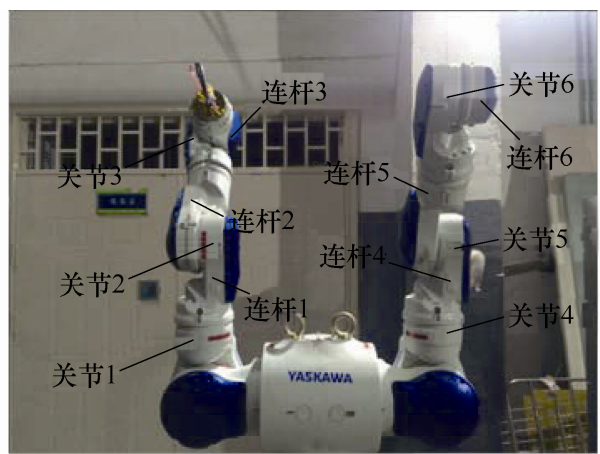

(a)初始状态

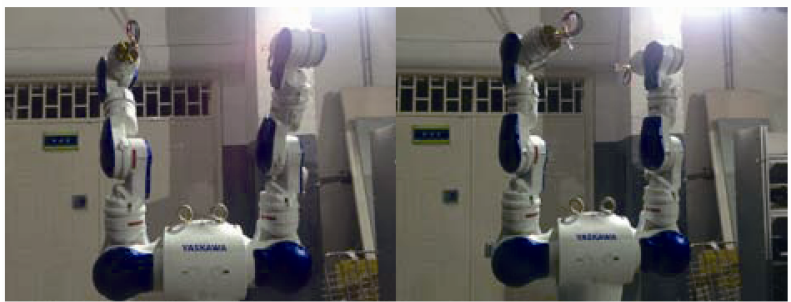

(b)1s时状态

(c) 2 s时状态

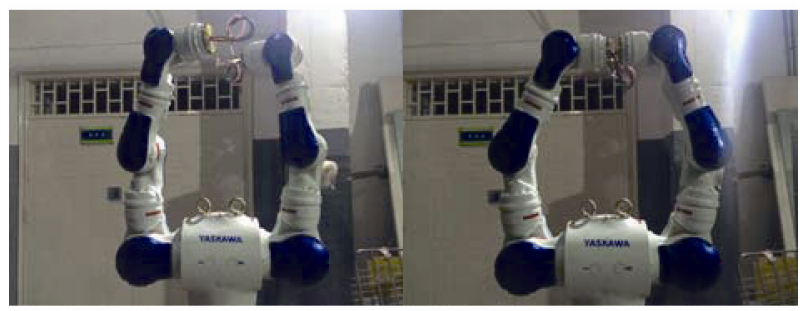

(d)3s时状态

(e) 3.75 s 时状态

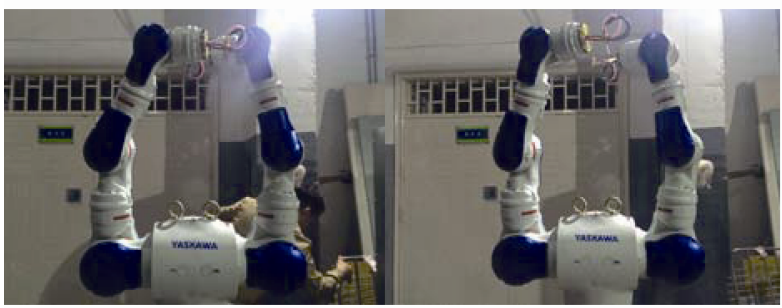

(f) 4 s时状态

(g) 5 s 时状态

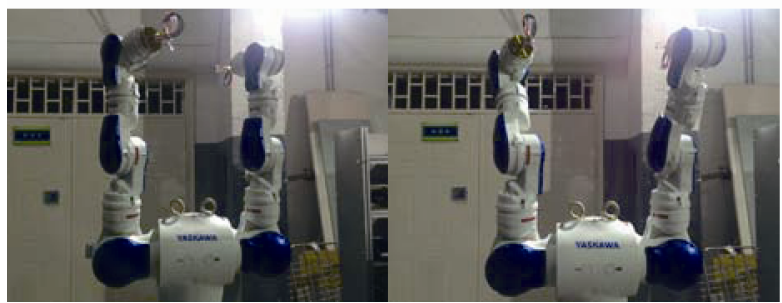

(h)6s时状态

(i) $7 \mathrm{~s}$ 时状态

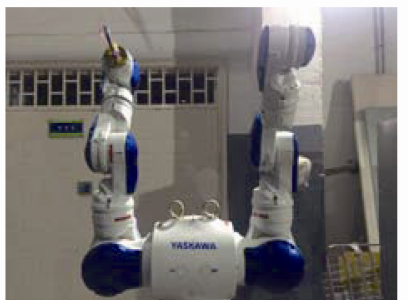

(j) 7.5 s时状态

图 10 安川双臂机器人 SDA10F 试验

在 Matlab2014 中设定两机械臂关节和连杆参 数, 定义各矩阵及其中元素, 再编辑相对动力学表 达式(18), 运行得到两臂末端相对力理论值曲线。 安川双臂机器人 SDA10F 各关节运动参数根据图 $3 \sim 8$ 设定, 运行过程中通过开放的数据端口连接电 脑采集两臂末端相对力实际值, 并将数据导入 Excel 制成曲线，与理论值对比。

两机械臂末端相对作用力及力矩仿真和试验对 比结果如图 11 14 所示。机械臂关节转动分加速 段、匀速段和减速段, 到达目标点后停止, 再重复 上述动作回到起点, 完成一周循环, 时间为 $7.5 \mathrm{~s}$ 。 图 11 14 为三个周期运动情况, 总时间为 $22.5 \mathrm{~s}$ 。

以图 12 为例分析: 如标记 3 所示, 此时, 关节 减速、停止再加速运动, 相对力理论值趋势变化明 显。实际值曲线上标记 4 和 5 对应标记 3 中关节减 速和加速, 标记 4 为关节减速导致相对力产生变小 


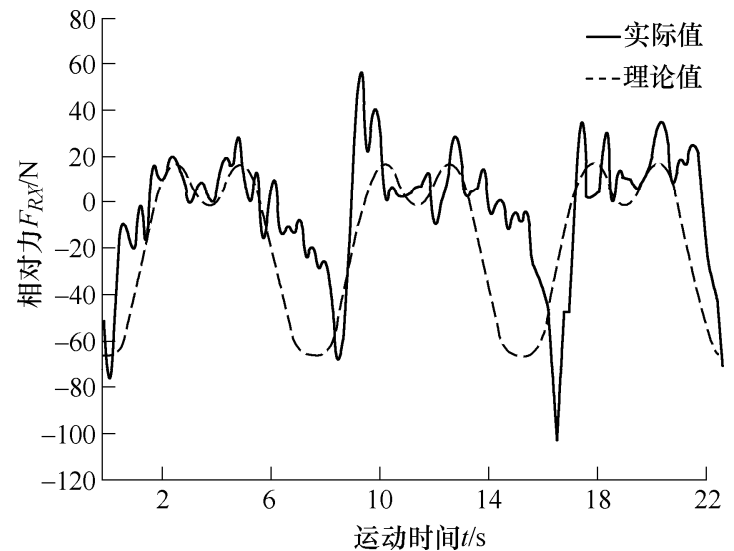

图 11 两机械臂末端 $X$ 轴方向相对力 $F_{R X}$

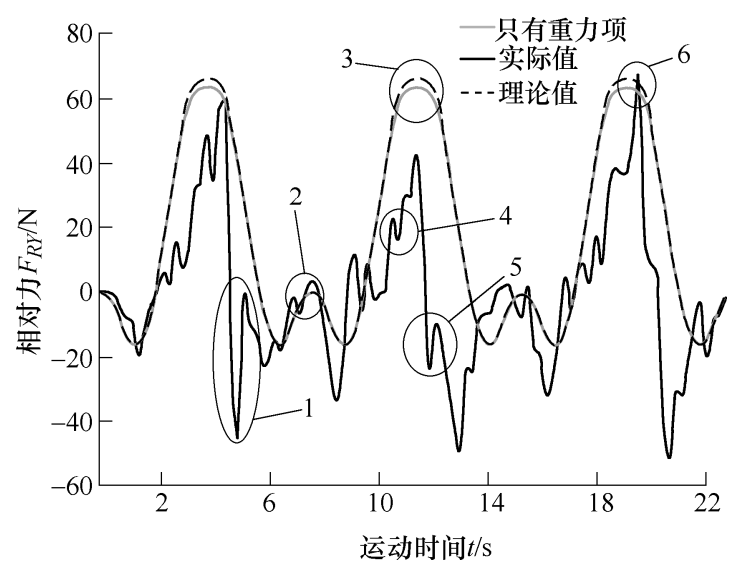

图 12 两机械臂末端 $Y$ 轴方向相对力 $F_{R Y}$

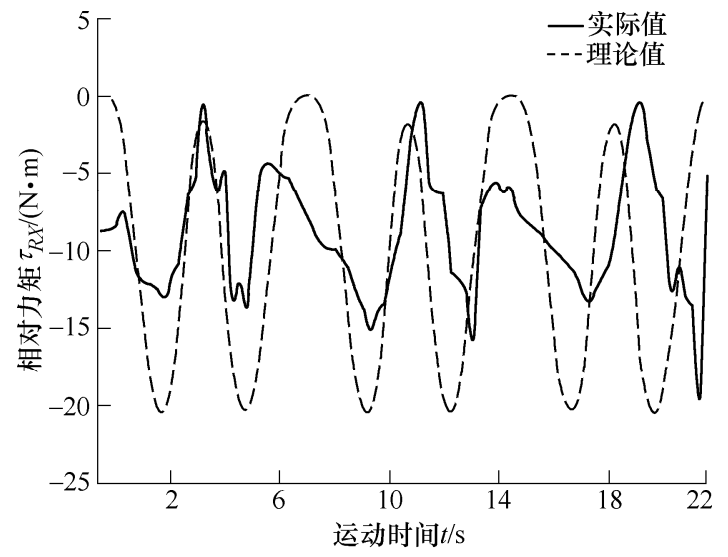

图 13 两机械臂末端 $X$ 轴方向相对力矩 $\tau_{R X}$

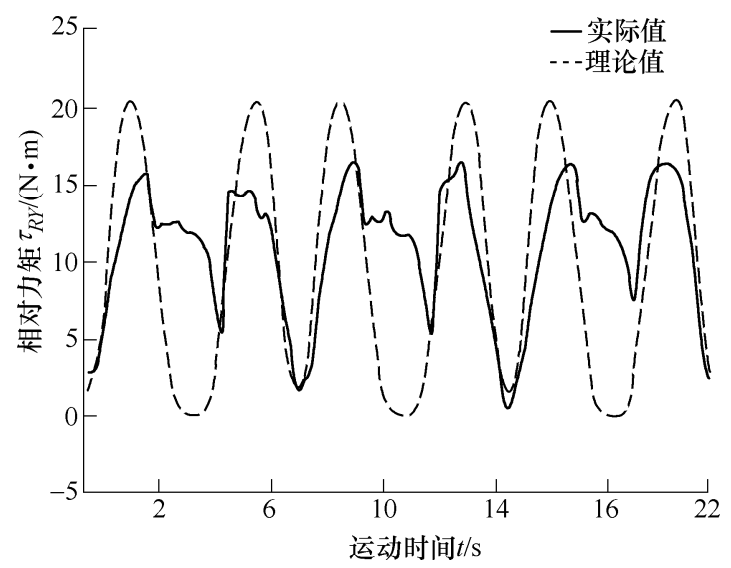

图 14 两机械臂末端 $Y$ 轴方向相对力矩 $\tau_{R Y}$
波动, 对应标记 3 中趋势变化减缓, 标记 5 为关节 加速导致相对力产生变大波动, 对应标记 3 中趋势 变化增强。关节加速和减速时, 相对力和力矩的实 际值无法像理论值一样平滑, 有明显波动, 与理论 值有一定误差。两机械臂到目标点停止再回到起点 循环运动, 实际上, 到达目标点和回到起点时有短 暂停止, 和启动时情况相似, 相对力实际值会有明 显突变, 如标记 6 所示, 形成尖角。标记 1 时刻关 节加速度消失, 此时两臂末端各自运动轨迹都偏向 惯性坐标系 $Y$ 轴方向, 末端作用力在 $Y$ 轴上分量远 大于 $X$ 轴, 相对力 $F_{R Y}$ 才会产生这么明显的突变。 标记 2 时刻两臂末端各自运动轨迹都偏向惯性坐标 系 $X$ 轴方向, 所以 $Y$ 轴方向相对力波动不明显。

由整体仿真与试验分析可得以下结果。

(1) 试验中, 两机械臂始终关于底座坚直轴线 中心对称运动, 其末端在惯性坐标系 $Z$ 轴方向运动 状态一致, 各时刻位置、速度和加速度参数相同, 所以两臂末端力和力矩大小和方向相同, 求相对力 $F_{R Z}$ 和相对力矩 $\tau_{R Z}$ 时结果为零。

(2) 通过仿真与试验数据对比发现, 当两机械 臂末端相对力 $\boldsymbol{F}_{R}=\left(\boldsymbol{J}_{R}^{\mathrm{T}}\right)^{+}[\boldsymbol{M}(\boldsymbol{q}) \ddot{\boldsymbol{q}}+\boldsymbol{H}(\boldsymbol{q}, \dot{\boldsymbol{q}})+\boldsymbol{G}(\boldsymbol{q})]$ 只保留重力项, 变为 $\boldsymbol{F}_{R}=\left(\boldsymbol{J}_{R}^{\mathrm{T}}\right)^{+} \boldsymbol{G}(\boldsymbol{q})$ 时, 如图 12 中 只有重力项所示, 与相对力理论值曲线基本相同, 而且与实际值变化趋势基本吻合。其原因是：两机 械臂末端在坚直方向相对运动时, 关节驱动力矩 $\boldsymbol{\tau}$ 需克服重力做功, 对应末端相对力表达式中重力项 部分 $\boldsymbol{G}(\boldsymbol{q})$, 虽然关节速度和加速度对两臂末端相对 力有一定作用, 使力和力矩曲线有波动变化, 但是 无法明显改变力和力矩变化趋势, 只有较小影响, 起决定作用的是重力项, 两臂末端主要是克服重力 相对运动。

(3) 建立相对动力学模型时所用机械臂关节和 连杆参数与实际值有一定误差, 且该模型在计算系 统动能和势能时为计算方便有一定简化, 无法与实 际完全一致, 而且模型中没有摩擦项, 没有考虑摩 擦等干扰因素的影响, 所以仿真数据与实际数据有 一定误差。但是通过图 11 14 实际值与理论值对比 分析, 无论 $X 、 Y$ 轴方向, 力和力矩理论值与实际 值都是对应波动变化, 其产生原因也相同, 依据相 对动力学模型得到的理论值与实际采集的相对力和 力矩变化趋势和范围是一致的, 可以证明相对动力 学模型的正确性。

\section{3 结论}

(1) 本文使用矢量解析法求解角速度雅可比矩 
阵, 与微分变换法对比表明, 矢量解析法求解过程 清晰形象，避免复杂计算，与微分变换法求解结果 相同。

(2) 双臂协调机器人相对动力学模型能够建立 两机械臂末端相对作用力及力矩与各关节参数之间 联系，使双臂协调不局限于夹持目标物体，形成闭 链系统，为分析双臂协调运动提供理论依据。

\section{参 考 文 献}

[1] TAO J M, LUH J Y S. Robust position and force control for a system of multiple redundant-robots[C]// Proceedings of the 1992 IEEE International Conference on Robotics and Automation, Nice, France-May, 1992: 2211-2216.

[2] 程靖, 陈力. 空间机器人双臂捕获卫星力学分析及镇定 控制[J]. 力学学报, 2016，48(4): 832-842.

CHENG Jing, CHEN Li. Mechanical analysis and calm control of dual-arm space robot for capturing a satellite[J]. Chinese Journal of Theoretical and Applied Mechanics, 2016, 48(4): 832-842.

[3] 董楸煌, 陈力. 双臂空间机器人捕获非合作目标冲击效 应分析及闭链混合系统力/位形鲁棒镇定控制[J]. 机械 工程学报, 2015, 51(9): 37-44.

DONG Qiuhuang, CHEN Li. Impact effect analysis of dual-arm space robot capturing a non-cooperative target and force/position robust stabilization control for closed-chain hybrid system[J]. Journal of Mechanical Engineering, 2015, 51(9): 37-44.

[4] JIA Y H, HU Q, XU S J. Dynamics and adaptive control of a dual-arm space robot with closed-loop constraints and uncertain inertial parameters[J]. Acta Mechanica Sinica, 2014, 30(1): 112-124.

[5] GE X, JIN J. Dynamics analyze of a dual-arm space robot system based on Kane's method[C]// International Conference on Industrial Mechatronics and Automation. IEEE，2010: 646-649.

[6] 刘佳, 刘荣. 双臂协调机械手动力学建模的新方法 [J]. 北京航空航天大学学报, 2016 , 42 (9): 1903-1910.

LIU J, LIU Rong. New approach for dynamics modeling of dual-arm cooperating manipulators[J]. Journal of Beijing University of Aeronautics and Astronautics, 2016, 42(9): 1903-1910.

[7] JAMISOLA R S, KORMUSHEV P S, ROBERTS R G, et al. Task-space modular dynamics for dual-arms expressed through a relative Jacobian[J]. Journal of Intelligent \& Robotic Systems， 2016， 83(2): 205-218.

[8] SHIN S Y, LEE J W, KIM C H. Humanoid's dual arm object manipulation based on virtual dynamics model[C]// IEEE International Conference on Robotics and Automation. IEEE，2012: 2599-2604.

[9] 蔡自兴, 谢斌. 机器人学 $[M]$. 北京: 清华大学出版社, 2015. CAI Zixing, XIE Bin. Robotics[M]. Beijing: Tsinghua University Press, 2015

[10] XI W. Study of new analytic solution of robotic relative Jacobian matrix[J]. Journal of Southeast University, 2002, 32(4): 614-619.

[11] LEWIS C L. Trajectory generation for two robots cooperating to perform a task[C]// IEEE International Conference on Robotics and Automation , 1996. Proceedings. IEEE， 1995: 1626-1631.

[12] LEWIS C L, MACIEJEWSKI A A. Trajectory generation for cooperating robots[C]// IEEE International Conference on Systems Engineering. IEEE， 2012: 300-303.

[13] JAMISOLA R S, ROBERTS R G. A more compact expression of relative Jacobian based on individual manipulator Jacobians[M]. North-Holland Publishing Co. 2015.

[14] SICILIANO B, SCIAVICCO L, VILLANI L, et al. Robotics: Modelling, planning and control[J]. Advanced Textbooks in Control \& Signal Processing, 2009, 4(12): 76-82.

作者简介：张建华，男，1979 年出生，博士，教授，博士研究生导师。 主要研究方向为智能机器人技术与超精密加工。

E-mail: jhzhang@hebut.edu.cn

许晓林, 男, 1992 年出生, 硕士研究生。主要研究方向为智能机器 人技术。

E-mail: 13512287970@163.com

刘璇(通信作者), 女, 1980 年出生, 博士, 讲师。主要研究方向为智能 机器人技术。

E-mail: xuaner1007@163.com 